\title{
Transient Stability Analysis by Reachable Set Computation
}

\author{
Matthias Althoff, Miloš Cvetković, and Marija Ilić
}

\begin{abstract}
We present a formal technique for verifying the stability of transient responses of power systems. The procedure uses reachability analysis to compute the complete set of possible transient responses starting from a set of initial states, subject to a dynamics specified by differential-algebraic equations. The method is constructive and fully automatic, two properties that are often hard to achieve with direct Lyapunov methods when the differential-algebraic equations are not simplified. Reachability analysis is computationally expensive, but this work presents new techniques that make it possible to verify the stability of a transient response of the IEEE 14-bus benchmark power system network.
\end{abstract}

Index Terms-Transient Stability, Reachability Analysis, Nonlinear Differential-Algebraic Equations, Power Systems

\section{INTRODUCTION}

Transient stability analysis of power systems goes back to the 1920s [10]. Since then, many approaches for transient stability analysis have been developed [21]. We group the techniques into model-based and model-free approaches. Model-free approaches predict the transient stability based on machine learning techniques, where Neural Nets [9] and pattern recognition [22] are most popular.

Since we propose a model-based technique, we focus the literature review on this category. The most common modelbased technique is to simulate power system equations by numerical integration. The main advantage of numerical integration is its versatility, meaning that all kinds of models can be analyzed, while the main disadvantage is the limited applicability in emergency situations, when the consequences of a fault have to be known immediately. For this reason, approaches for the parallelization of numerical integration are researched [2], [25]. Alternatively, Monte-Carlo simulation provides a probabilistic evaluation, where many scenarios are deterministically computed and later evaluated by their probability of occurrence. Aggregation of all results yields a probability that the transient response is unstable [8].

In order to improve the online analysis during a faulton situation, direct methods based on the Lyapunov stability theory have been developed [1], [12], [23]. The main advantage of Lyapunov techniques is that one can guarantee that the transient response is stable when the post-fault state is within a previously computed domain of attraction. The main disadvantage is that the region of attraction is usually

Matthias Althoff is with Faculty of Computer Science and Automation, Ilmenau University of Technology, 98693 Ilmenau, Germany, email: matthias.althoff@tu-ilmenau.de

Miloš Cvetković and Marija Ilić are with Faculty of Electrical and Computer Engineering, Carnegie Mellon University, Pittsburgh, PA 15213, USA, email: mcvetkoveandrew. cmu.edu, milic@ece.cmu.edu conservative, i.e. largely underapproximated when the system is relatively large. For practical applications, Lyapunov-based models are computationally infeasible, or one has to drastically simplify the system dynamics, resulting in possibly incorrect results [21].

Recently, reachability analysis as a new kind of analysis technique has emerged. Reachability analysis combines advantages of numerical integration and Lyapunov-based techniques. Reachability analysis computes the set of all possible trajectories of a system, given a set of initial states, a set of disturbances, and a set of uncertain parameters. Thus, one obtains a set-based evolution of the system dynamics, similar to a numerical integration, except that all possible solutions are computed for each time interval at once. Due to the set-based computation, the result is rigorous as for Lyapunov-based analysis. One can prove transient stability without constructing a Lyapunov function by showing that the system returns to the set of initial states after a fault occurred. The main disadvantage of reachability analysis so far was the computational complexity for power system applications, so that only small systems have been verified [13], [19], [26]. In [19], transient stability analysis is performed using level-sets for a single-machine-infinite-bus system modeled by ODEs with only 2 state variables. A slightly larger double-machineinfinite-bus system with 2 buses described by ODEs with 5 state variables is considered in [26]. In [13], an initial DAE model is simplified to ODEs and further to linear ODEs, without considering errors made during each conversion. A 3-bus system is considered in [13], and effects on wind variability rather than transient stability are investigated.

In this work, we present a new approach for reachable set computation, which is much more scalable than previous approaches and additionally guarantees that the result is overapproximative. This property is important for proving transient stability. The approach works for any kind of system with time-invariant, semi-explicit, index-1 differentialalgebraic equations (DAEs). We show the scalability by computing the reachable set for the IEEE 14-bus benchmark power system network to which we add 5 generators, resulting in 14 differential and 28 algebraic variables, giving a total of 42 continuous state variables.

The main reasons for the improved scalability is because we (i) invented a new and efficient approach to tightly overapproximate the complicated nonlinear DAEs by linear differential inclusions, and (ii) apply zonotopes for the reachability computation of the linear differential inclusions, which outperform all previous approaches for this system class [15]. 


\section{Problem Statement}

Power systems can be formalized as a set of semi-explicit, nonlinear index-1 DAEs (see e.g. [24]). We assume that the parameters of the power system are known and constant over time, resulting in a set of time-invariant DAEs. We introduce the vectors of differential variables as $x \in \mathbb{R}^{n_{d}}$, algebraic variables as $y \in \mathbb{R}^{n_{a}}$, and inputs as $u \in \mathbb{R}^{m}$, where $n_{d}, n_{a}$, and $m$ are the number of differential, algebraic, and input variables, respectively. Further, we introduce the set of consistent initial states $\mathcal{R}(0)$ and the set of possible inputs/disturbances $\mathcal{U}$. The set of DAEs can now be written as

$$
\begin{gathered}
\dot{x}=f(x(t), y(t), u(t)) \\
0=g(x(t), y(t), u(t)), \\
{\left[x^{T}(0), y^{T}(0)\right]^{T} \in \mathcal{R}(0), \quad u(t) \in \mathcal{U},}
\end{gathered}
$$

The initial state is consistent when $g(x(0), y(0), u(0))=0$ and we assume that (1) has a unique solution $\gamma(t, x(0), y(0), u(\cdot))$ for all consistent initial states $x(0), y(0)$ and all piecewise continuous input trajectories $u(\cdot)$, where $u(t)$ refers to an input at a specific point in time $t$. We are interested in computing the reachable set of (1) for a time interval $\left[0, t_{f}\right]$, which is defined as

$$
\begin{aligned}
\mathcal{R}^{e}\left(\left[0, t_{f}\right]\right)=\{ & \gamma(t, x(0), y(0), u(\cdot)) \mid\left[x^{T}(0), y^{T}(0)\right]^{T} \in \mathcal{R}(0), \\
& \left.u(t) \in \mathcal{U}, t \in\left[0, t_{f}\right]\right\} .
\end{aligned}
$$

The superscript $e$ on $\mathcal{R}^{e}\left(\left[0, t_{f}\right]\right)$ denotes the exact reachable set, which cannot be computed for nonlinear DAE systems [20]. For this reason, we aim to compute overapproximations $\mathcal{R}\left(\left[0, t_{f}\right]\right) \supseteq \mathcal{R}^{e}\left(\left[0, t_{f}\right]\right)$, which are as accurate as possible, while at the same time ensuring that the computations are efficient and scale well with the system dimension $n=n_{d}+n_{a}$. For simplification we often use reachable set instead of always emphasizing the we compute overapproximative reachable sets. If the overapproximation shows transient stability, we can conclude that the exact result is stable since all solutions of the real system are included in the overapproximation. The projection of the reachable set onto the differential variables is denoted by $\mathcal{R}^{d}\left(\left[0, t_{f}\right]\right)$ and for the algebraic variables by $\mathcal{R}^{a}\left(\left[0, t_{f}\right]\right)$.

In this work, we continue to compute reachable sets until a time $t_{f}$ for which $\mathcal{R}\left(t_{f}\right) \subseteq \mathcal{R}(0)$, i.e. all solutions are within the set of initial states such that we can conclude transient stability of the system in the sense that all transient responses return to the set of initial states. Using the same method, one could also check if an arbitrarily small region around the steady state can be reached. As a by-product, we obtain all voltage and phase limits over time for further analysis.

\section{MAIn AlgORITHM}

The reachable set computation is performed by conservatively simplifying the nonlinear DAEs to ordinary linear differential inclusions

$$
\dot{\tilde{x}} \in \tilde{A} \tilde{x} \oplus \tilde{\mathcal{U}}
$$

where the derivative is not exactly known, but bounded by a set. In (2), we use a set-based addition (Minkowski addition), which we introduce together with the set-based multiplication:

$$
\begin{aligned}
& \mathcal{A} \oplus \mathcal{B}:=\{a+b \mid a \in \mathcal{A}, b \in \mathcal{B}\}, \\
& \mathcal{A} \otimes \mathcal{B}:=\{a b \mid a \in \mathcal{A}, b \in \mathcal{B}\} .
\end{aligned}
$$

The set of added inputs $\tilde{\mathcal{U}}$ in (2) is chosen such that it includes all behaviors of the differential variables of the original nonlinear DAE-system. The solution of the algebraic variables will be obtained in a subsequent computation. We construct different differential inclusions for fixed time intervals $t \in \tau_{k}:=\left[t_{k}, t_{k+1}\right]$, where $t_{k}=k r, k \in \mathbb{N}$ is the time step and $r \in \mathbb{R}^{+}$is referred to as the time increment or step size. An extension to variable step sizes is described in [14].

The reachable set computation itself is performed on the differential inclusion overapproximation in (2) for which efficient algorithms exist when using zonotopes [15], [17] or support functions [16] as representations of the reachable set. We use zonotopes since some operations required for the conversion to linear differential inclusions, such as quadratic maps, can be efficiently computed with them.

In the remainder of this section, we describe the main algorithm in Fig. 1 in words. Details of the algorithm are described in the subsequent sections. We first linearize the differential and algebraic equations of (1) using a first order Taylor expansion. Next, we heuristically obtain a set of linearization errors $\overline{\mathcal{L}}^{d}$ and $\overline{\mathcal{L}}^{a}$ for the differential and algebraic equations, which we believe to include the actual set of linearization errors. The combination of linearized equations to which the set of linearization errors is added, is referred to as conservatively linearized equations. After inserting the conservatively linearized algebraic equations into the conservatively linearized differential equations, we obtain a differential inclusion of the form in (2). The reachable set computation of (2) returns the set $\overline{\mathcal{R}}^{d}\left(\tau_{k}\right)=\mathcal{R}_{\text {affine }}^{d}\left(\tau_{k}\right) \oplus \overline{\mathcal{R}}_{p}^{d}(r)$ for the $k^{\text {th }}$ time interval, which is composed of the affine solution $\mathcal{R}_{\text {affine }}^{d}\left(\tau_{k}\right)$ (no uncertain inputs) and the solution due to uncertain inputs $\tilde{\mathcal{U}}$. This reachable set is then used to overapproximately determine the linearization errors $\mathcal{L}^{d}$ and $\mathcal{L}^{a}$ of the differential and algebraic equations, respectively. If $\mathcal{L}^{d} \nsubseteq \overline{\mathcal{L}}^{d}$ or $\mathcal{L}^{a} \nsubseteq \overline{\mathcal{L}}^{a}$, one or both of the assumed sets $\overline{\mathcal{L}}^{d}$ and $\overline{\mathcal{L}}^{a}$ have to be enlarged. When $\mathcal{L}^{d} \subseteq \mathcal{L}_{\text {max }}^{d}$ or $\mathcal{L}^{a} \subseteq \mathcal{L}_{\text {max }}^{a}$, where $\mathcal{L}_{\text {max }}^{d}$ and $\mathcal{L}_{\text {max }}^{a}$ are user-defined bounds for linearization errors, the reachable set has to be split to reduce the linearization error. As for all subdivision methods, splitting leads to improved accuracy, but increases the computational demand. Finally, we compute the reachable set due to uncertain inputs $\mathcal{R}_{p}^{d}(r)$ based on $\mathcal{L}^{d}$ and $\mathcal{L}^{a}$ (instead of $\overline{\mathcal{L}}^{d}$ and $\overline{\mathcal{L}}^{a}$ ), which tightens the reachable set to $\mathcal{R}^{d}\left(\tau_{k}\right)=\mathcal{R}_{\text {affine }}^{d}\left(\tau_{k}\right) \oplus \mathcal{R}_{p}^{d}(r)$. The computation continues with the next time interval.

\section{CONSERVATIVE LINEARIZATION}

In this section we describe how we overapproximate the DAEs to linear differential inclusions. Thereto, we introduce the vector $z=\left[x^{T}, y^{T}, u^{T}\right]^{T}$, the linearization point $z^{*}=$ $\left[x^{* T}, y^{* T}, u^{* T}\right]^{T}$, and $\mathcal{R}^{z}=\mathcal{R}\left(\tau_{k}\right) \times \mathcal{U}$. 
Initial set $\mathcal{R}(0)$, time step $k=1$, sets of allowed linearization error $\overline{\mathcal{L}}^{d}, \overline{\mathcal{L}}^{a}$

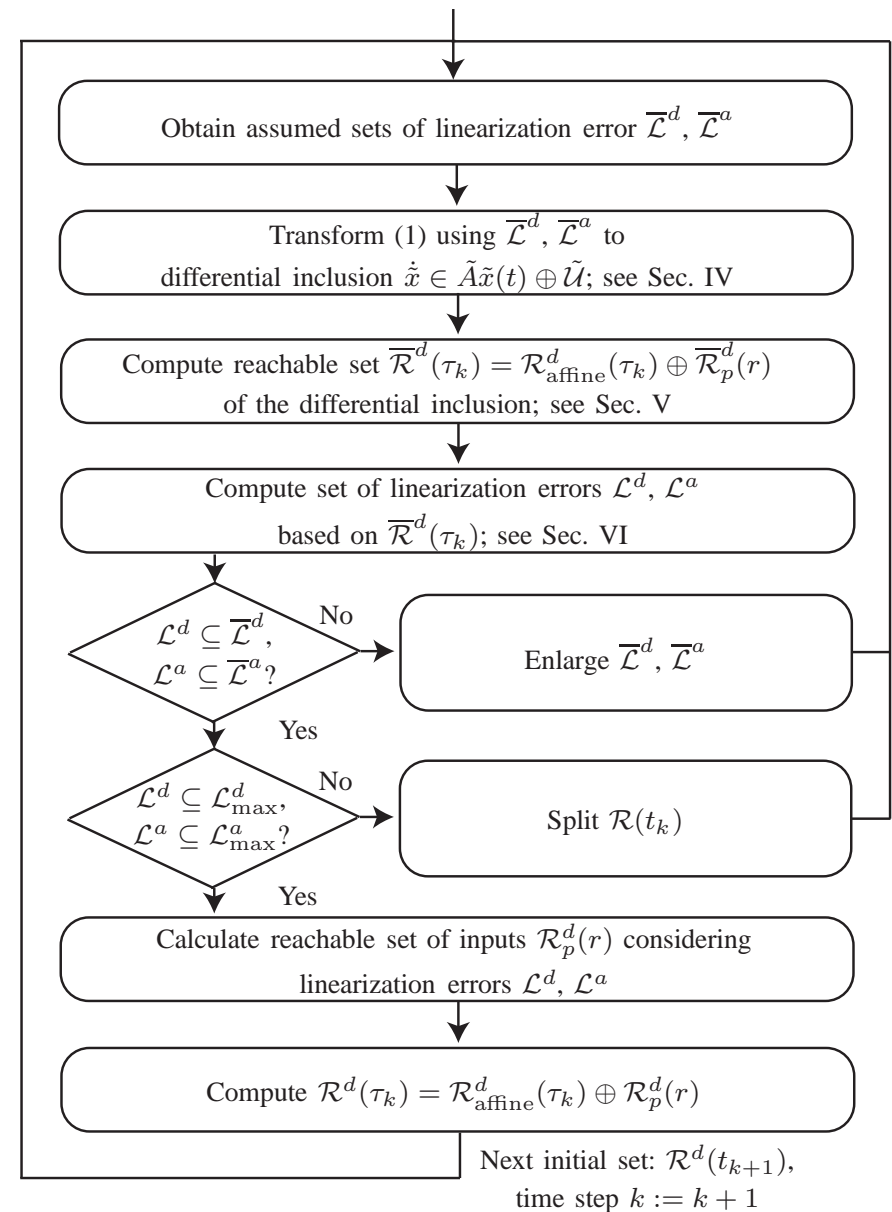

Fig. 1. Overview of reachable set computation.

Before we perform the linearization, we have to choose the linearization point $z^{*}$ (which varies for each time interval). In order to reduce the linearization error, we use $z^{*}$ as the center of $\mathcal{R}^{z}\left(\tau_{k}\right)$ of the currently computed time interval $\tau_{k}$, see [7]. We approximate the center of the yet unknown set $\mathcal{R}^{z}\left(\tau_{k}\right)$ by $x^{*}\left(\tau_{k}\right)=c^{d}\left(t_{k}\right)+0.5 f\left(c^{d}, c^{a}, c^{u}\right)\left(t_{k+1}-t_{k}\right)$, where $c^{d}, c^{a}, c^{u}$ are the volumetric centers of the sets $\mathcal{R}^{d}\left(t_{k}\right)$, $\mathcal{R}^{a}\left(t_{k}\right)$, and $\mathcal{U}$. This procedure can be interpreted as a onestep Euler integration. We further choose $u^{*}\left(\tau_{k}\right)=c^{u}$ and the linearization point of the algebraic part is obtained by solving $0=g\left(x^{*}, y^{*}, u^{*}\right)$ using a Newton-Raphson algorithm.

Using $z^{*}$, the linearization of (1) is performed by a firstorder Taylor expansion with Lagrangian remainder:

$$
\begin{gathered}
\dot{x}_{i}=f_{i}(z(t)) \in f_{i}\left(z^{*}\right)+\left.\frac{\partial f_{i}(z)}{\partial z}\right|_{z=z^{*}}\left(z(t)-z^{*}\right) \oplus \mathcal{L}_{i}^{d}, \\
0=g_{j}(z(t)) \in g_{j}\left(z^{*}\right)+\left.\frac{\partial g_{j}(z)}{\partial z}\right|_{z=z^{*}}\left(z(t)-z^{*}\right) \oplus \mathcal{L}_{j}^{a},
\end{gathered}
$$

where

$\mathcal{L}_{i}^{d}=\left\{\left.\frac{1}{2}\left(z(t)-z^{*}\right)^{T} \frac{\partial^{2} f_{i}(z)}{\partial z^{2}}\right|_{z=\xi}\left(z(t)-z^{*}\right) \mid \xi \in \mathcal{R}^{z}, z(t) \in \mathcal{R}^{z}\right\}$

and $\mathcal{L}_{j}^{a}$ is computed analogously by replacing $f_{i}(z)$ with $g_{j}(z)$. We write $\mathcal{L}_{i}^{d}$ for the projection of $\mathcal{L}^{d}$ onto the $i^{\text {th }}$ coordinate. The Lagrangian remainders $\mathcal{L}^{d}, \mathcal{L}^{a}$ enclose all higher-order terms if $\xi$ can take any value of the linear combination of $z$ and $z^{*}$, i.e. $\xi \in\left\{\alpha z+(1-\alpha) z^{*} \mid \alpha \in[0,1]\right\}$, which follows from the mean value theorem [11, p. 87]. Since (i) $z(t)$ can take any values from $\mathcal{R}^{z}$ in the time interval $\tau_{k}$, (ii) $\mathcal{R}^{z}$ is represented by a convex zonotope, and (iii) $z^{*}$ is chosen as an interior point of this set, it follows that for $\xi \in \mathcal{R}^{z}$ the set of Lagrangian remainders is captured.

For subsequent derivations, we introduce the Jacobians of $f(z(t))$ with respect to $x, y, u$, which are $A \in \mathbb{R}^{n_{d} \times n_{d}}, B \in$ $\mathbb{R}^{n_{d} \times m}$, and $C \in \mathbb{R}^{n_{d} \times n_{a}}$, where $\left.\frac{\partial f_{i}(z)}{\partial z}\right|_{z=z^{*}}=\left[\begin{array}{lll}A & C & B\end{array}\right]$. The Jacobians for $g(z(t))$ are $D \in \mathbb{R}^{n_{a} \times n_{d}}, E \in \mathbb{R}^{n_{a} \times m}$, and $F \in \mathbb{R}^{n_{a} \times n_{a}}$, where $\left.\frac{\partial g_{j}(z)}{\partial z}\right|_{z=z^{*}}=\left[\begin{array}{lll}D & F & E\end{array}\right]$. We further introduce the Hessians $\left.H^{d,(i)}(\xi)=\frac{\partial^{2} f_{i}(z)}{\partial z^{2}}\right)\left.\right|_{z=\xi}$ and $\left.H^{a,(j)}(\xi)=\frac{\partial^{2} g_{j}(z)}{\partial z^{2}}\right)\left.\right|_{z=\xi}$, as well as the following variables with respect to the linearization point: $\Delta x(t)=x(t)-x^{*}$, $\Delta u(t)=u(t)-u^{*}, \Delta y(t)=y(t)-y^{*}, \nu(t)=z(t)-z^{*}$, and $\mathcal{R}_{\Delta}^{z}=\mathcal{R}^{z} \oplus\left(-z^{*}\right)$. Using the previously introduced variables, we have from (3) and (4) that

$$
\begin{aligned}
& \dot{x} \in f\left(z^{*}\right)+A \Delta x(t)+B \Delta u(t)+C \Delta y(t) \\
& \oplus\left\{\frac{1}{2} \sigma \mid \sigma_{i}=\nu^{T} H^{d,(i)}(\xi) \nu, \xi \in \mathcal{R}^{z}, \nu \in \mathcal{R}_{\Delta}^{z}\right\}, \\
& 0 \in g\left(z^{*}\right)+D \Delta x(t)+E \Delta u(t)+F \Delta y(t) \\
& \oplus\left\{\frac{1}{2} \phi \mid \phi_{j}=\nu^{T} H^{a,(j)}(\xi) \nu, \xi \in \mathcal{R}^{z}, \nu \in \mathcal{R}_{\Delta}^{z}\right\} .
\end{aligned}
$$

Next, we reformulate (6) to

$$
\begin{gathered}
\Delta y(t) \in-F^{-1}\left(g\left(z^{*}\right)+D \Delta x(t)+E \Delta u(t)\right) \\
\oplus\left\{-\frac{1}{2} F^{-1} \phi \mid \phi_{j}=\nu^{T} H^{a,(j)}(\xi) \nu, \xi \in \mathcal{R}^{z}, \nu \in \mathcal{R}_{\Delta}^{z}\right\} .
\end{gathered}
$$

Note that $F$ is always invertible due to the index-1 property of the DAEs. Inserting (7) into (5) results in a differential inclusion

$$
\begin{aligned}
\dot{x} \in & f\left(z^{*}\right)+A \Delta x(t)+B \Delta u(t) \\
& -C F^{-1}\left(g\left(z^{*}\right)+D \Delta x(t)+E \Delta u(t)\right) \oplus \mathcal{L} \\
= & (k+\tilde{A} \Delta x(t)+\tilde{B} \Delta u(t)) \oplus \mathcal{L},
\end{aligned}
$$

where

$$
\begin{aligned}
& k=f\left(z^{*}\right)-C F^{-1} g\left(z^{*}\right) \\
& \tilde{A}=A-C F^{-1} D \\
& \tilde{B}=B-C F^{-1} E .
\end{aligned}
$$

and

$$
\begin{aligned}
\mathcal{L}= & \left\{\frac{1}{2}\left(\sigma-C F^{-1} \phi\right) \mid \sigma_{i}=\nu^{T} H^{d,(i)}(\xi) \nu,\right. \\
& \left.\phi_{j}=\nu^{T} H^{a,(j)}(\xi) \nu, \xi \in \mathcal{R}^{z}, \nu \in \mathcal{R}_{\Delta}^{z}\right\} \\
\subseteq & \mathcal{L}^{d} \oplus\left(-C F^{-1} \otimes \mathcal{L}^{a}\right)
\end{aligned}
$$

The set of linearization errors $\mathcal{L}$ as proposed in (9) is a subset of $\mathcal{L}^{d} \oplus\left(-C F^{-1} \otimes \mathcal{L}^{a}\right)$ since in the latter computation, the dependency of $\nu$ is ignored when computing $\mathcal{L}^{d}$ and $\mathcal{L}^{a}$ separately. We can further simplify the reachable set computation of (8) by solving

$$
\begin{gathered}
\dot{\tilde{x}} \in \tilde{A} \tilde{x}(t) \oplus \tilde{\mathcal{U}} \\
\tilde{x}(t)=\Delta x(t), \quad \tilde{\mathcal{U}}:=k \oplus \tilde{B}\left(\mathcal{U} \oplus\left(-u^{*}\right)\right) \oplus \mathcal{L} .
\end{gathered}
$$


The problem of the above system is that the set of linearization errors $\mathcal{L}$ is not known in advance, so $\tilde{\mathcal{U}}$ is unknown, too. For this reason, we have to guess the overapproximations $\overline{\mathcal{L}}^{d} \supseteq \mathcal{L}^{d}$ and $\overline{\mathcal{L}}^{a} \supseteq \mathcal{L}^{a}$. Using these two enclosures, it follows that $\overline{\mathcal{L}} \supseteq$ $\mathcal{L}$ for $\overline{\mathcal{L}}=\overline{\mathcal{L}}^{d} \oplus\left(-C F^{-1} \otimes \overline{\mathcal{L}}^{a}\right)$, see (9). As an initial guess we enlarge the previous linearization error by user-defined scalar factors $\lambda^{d} \in \mathbb{R}^{+}, \lambda^{a} \in \mathbb{R}^{+}$, so that

$$
\overline{\mathcal{L}}^{d}\left(\tau_{k}\right)=\hat{c}^{d} \oplus \lambda^{d}\left(\mathcal{L}^{d}\left(\tau_{k-1}\right) \oplus\left(-\hat{c}^{d}\right)\right),
$$

where $\hat{c}^{d}$ is the volumetric center of $\mathcal{L}^{d}\left(\tau_{k-1}\right)$, and $\overline{\mathcal{L}}^{a}\left(\tau_{k}\right)$ is obtained analogously. If it turns out that the enclosure assumption is not correct, the factors have to be repeatedly and automatically enlarged until the linearization error assumption holds. Further, if the sets $\overline{\mathcal{L}}^{d}$ and $\overline{\mathcal{L}}^{a}$ do not fulfill

$$
\overline{\mathcal{L}}^{d} \subseteq \mathcal{L}_{\max }^{d} \text { and } \overline{\mathcal{L}}^{a} \subseteq \mathcal{L}_{\max }^{a}
$$

where $\mathcal{L}_{\text {max }}^{d}$ and $\mathcal{L}_{\text {max }}^{a}$ are set by the user, the reachable set has to be split in order to reduce the linearization error until (11) is fulfilled. Thus, we can guarantee that the linearization error is bounded by $\mathcal{L}_{\text {max }}^{d}$ and $\mathcal{L}_{\text {max }}^{a}$. Techniques to decide the dividing hyperplane for the split can be found in [7].

\section{Reachable Set Computation of Linear Systems}

We briefly describe how the reachable set of a linear differential inclusion $\dot{\tilde{x}} \in \tilde{A} \tilde{x}(t) \oplus \overline{\mathcal{U}}$ (see (10)) with $\tilde{x}\left(t_{k}\right) \in \overline{\mathcal{R}}^{d}\left(t_{k}\right)$, is computed for the time interval $\tau_{k}$. We restrict the set of reachable sets $\overline{\mathcal{R}}^{d}\left(t_{k}\right)$ and inputs $\overline{\mathcal{U}}$ to zonotopes:

Definition 1 (Zonotope) Given a center $c \in \mathbb{R}^{n}$ and socalled generators $g^{(i)} \in \mathbb{R}^{n}$, a zonotope is defined as

$$
\mathcal{Z}=\left\{c+\sum_{i=1}^{p} \beta_{i} g^{(i)} \mid \beta_{i} \in[-1,1], c \in \mathbb{R}^{n}, g^{(i)} \in \mathbb{R}^{n}\right\}
$$

We write in short $\mathcal{Z}=\left(c, g^{(1)}, \ldots, g^{(p)}\right)$. A zonotope can be interpreted as the Minkowski addition of line segments $l^{(i)}=[-1,1] \mathrm{g}^{(i)}$, which is visualized step-by-step in a twodimensional vector space in Fig. 2. Zonotopes are a compact way of representing sets in high dimensions. More importantly, operations required for reachability analysis, such as linear maps $M \otimes \mathcal{Z}\left(M \in \mathbb{R}^{q \times n}\right)$ and Minkowski addition $\mathcal{Z}_{1} \oplus \mathcal{Z}_{2}$ can be computed efficiently and exactly, and others such as convex hull computation can be tightly overapproximated [15].

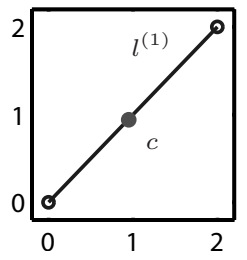

(a) $c \oplus l^{(1)}$

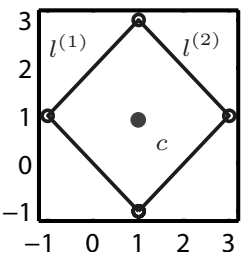

(b) $c \oplus l^{(1)} \oplus l^{(2)}$

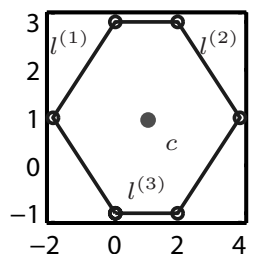

(c) $c \oplus \ldots \oplus l^{(3)}$
Fig. 2. Step-by-step construction of a zonotope.

As a preparation for the reachable set computation, we split the effect of $\overline{\mathcal{U}}$ into its center $\bar{u}_{c}$ and the translated set $\overline{\mathcal{U}}_{\Delta}=$ $\overline{\mathcal{U}} \oplus\left(-\bar{u}_{c}\right)$. This proposed reachable set computation takes advantage of the superposition principle for linear dynamics, as shown in Fig. 3:

1) Starting from $\overline{\mathcal{R}}^{d}\left(t_{k}\right)$, compute the set of all solutions $\mathcal{R}_{h}^{d}\left(t_{k+1}\right)$ for the affine dynamics $\dot{\tilde{x}}=\tilde{A} \tilde{x}(t)+\bar{u}_{c}$ at time $t_{k+1}$.

2) Obtain the convex hull of $\overline{\mathcal{R}}^{d}\left(t_{k}\right)$ and $\mathcal{R}_{h}^{d}\left(t_{k+1}\right)$ to approximate the reachable set for the time interval $\tau_{k}$.

3) Compute $\overline{\mathcal{R}}^{d}\left(\tau_{k}\right)$ by enlarging the convex hull to first bound all affine solutions within $\tau_{k}$ and secondly account for the set of uncertain inputs $\overline{\mathcal{U}}_{\Delta}$.

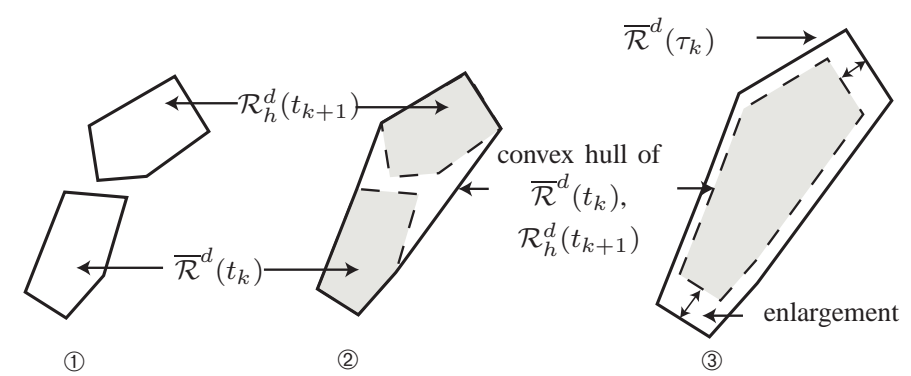

Fig. 3. Steps for the computation of an overapproximation of the reachable set for a linear differential inclusion.

Using $r=t_{k+1}-t_{k}$, the solution of $\mathcal{R}_{h}^{d}\left(t_{k+1}\right)$ is

$$
\mathcal{R}_{h}^{d}\left(t_{k+1}\right)=e^{\tilde{A} r} \overline{\mathcal{R}}^{d}\left(t_{k}\right)+\underbrace{\int_{0}^{r} e^{\tilde{A}(r-t)} d t \bar{u}_{c}}_{=: x_{p}(r)} .
$$

If $\tilde{A}$ is invertible, $x_{p}(r)$ can be computed as $\tilde{A}^{-1}\left(e^{\tilde{A} r}-I\right) \bar{u}_{c}$, where $I$ is the identity matrix. However, since $\tilde{A}$ is not always invertible, we compute $x_{p}(r)$ by integrating the Taylor series of $e^{\tilde{A} r}=\sum_{i=0}^{\infty}(\tilde{A} r)^{i} /(i !)$ :

$$
\begin{aligned}
x_{p}(r) & =(\sum_{i=0}^{\eta} \frac{\tilde{A}^{i} r^{i+1}}{(i+1) !}+\underbrace{\sum_{i=\eta+1}^{\infty} \frac{\tilde{A}^{i} r^{i+1}}{(i+1) !}}_{=: E_{p}(r)}) \bar{u}_{c} \\
& \in \underbrace{\left(\sum_{i=0}^{\eta} \frac{\tilde{A}^{i} r^{i+1}}{(i+1) !} \oplus \mathcal{E}_{p}(r)\right)}_{=: \Gamma(r)} \otimes \bar{u}_{c},
\end{aligned}
$$

The remainder $E_{p}(r)$ can be overapproximated by an interval matrix $E_{p}(r) \in \mathcal{E}_{p}(r):=[-W(r) r, W(r) r]$, i.e., by a matrix with lower and upper bounds on each element. Using symmetric bounds on $E_{p}(r)$, these bounds can be obtained from

$$
\begin{aligned}
\left|E_{p}(r)\right| & =\left|\sum_{i=\eta+1}^{\infty} \frac{\tilde{A}^{i}}{(i+1) !} r^{i+1}\right| \leq \sum_{i=\eta+1}^{\infty} \frac{|\tilde{A}|^{i} r^{i+1}}{(i+1) !} \\
& \leq\left(\sum_{i=\eta+1}^{\infty} \frac{|\tilde{A}|^{i} r^{i}}{i !}\right) r=\underbrace{\left(e^{|\tilde{A}| r}-\sum_{i=0}^{\eta} \frac{|\tilde{A}|^{i} r^{i}}{i !}\right)}_{=: W(r)} r .
\end{aligned}
$$

The enlargement of the convex hull denoted by $\overline{\mathcal{R}}_{\epsilon}^{d}$ to contain all affine solutions for $\tau_{k}$ is performed as in [3, Chap. 3.2]. 
The reachable set due to the uncertain and convex input $\overline{\mathcal{U}}_{\Delta}$ is obtained as derived in [6]:

$\overline{\mathcal{R}}_{p}^{d}(r)=\bigoplus_{i=0}^{\eta}\left(\frac{\tilde{A}^{i} r^{i+1}}{(i+1) !} \otimes \overline{\mathcal{U}}_{\Delta}\right) \oplus\left([-W(r) r, W(r) r] \otimes\left|\overline{\mathcal{U}}_{\Delta}\right|\right)$,

where the absolute value of a set of matrices $\mathcal{M}$ is defined elementwise as $|\mathcal{M}|_{i j}:=\sup \left\{\left|m_{i j}\right| \mid m \in \mathcal{M}\right\}$, which equivalently applies to the vector set $\overline{\mathcal{U}}_{\Delta}$.

The reachable set for the next point in time and time interval is obtained by combining all previous results and using the operator $\mathrm{CH}(\cdot)$ for the convex hull:

$$
\begin{aligned}
\overline{\mathcal{R}}^{d}\left(t_{k+1}\right) & =\underbrace{e^{A r} \overline{\mathcal{R}}^{d}\left(t_{k}\right) \oplus \Gamma(r) \bar{u}_{c}}_{=: \mathcal{R}_{\text {affine }}^{d}\left(t_{k+1}\right)} \oplus \overline{\mathcal{R}}_{p}^{d}(r), \\
\overline{\mathcal{R}}^{d}\left(\tau_{k}\right) & =\underbrace{\mathrm{CH}\left(\overline{\mathcal{R}}^{d}\left(t_{k}\right), e^{A r} \overline{\mathcal{R}}^{d}\left(t_{k}\right) \oplus \Gamma(r) \bar{u}_{c}\right) \oplus \overline{\mathcal{R}}_{\epsilon}^{d}}_{=: \mathcal{R}_{\text {affine }}^{d}\left(\tau_{k}\right)} \oplus \overline{\mathcal{R}}_{p}^{d}(r)
\end{aligned}
$$

Note that it is sufficient to add $\overline{\mathcal{R}}_{p}^{d}(r)$ for the time interval solution since $\overline{\mathcal{R}}_{p}^{d}(r)=\overline{\mathcal{R}}_{p}^{d}([0, r])$ when $\overline{\mathcal{U}}_{\Delta}$ contains the origin, causing monotone growth of $\overline{\mathcal{R}}_{p}^{d}(r)$ [3].

\section{COMPUtATION OF THE LinEARIZATION ERROR}

So far, we have computed an overapproximation of the reachable set of the dynamic variables $\overline{\mathcal{R}}^{d}\left(\tau_{k}\right)$ under the assumption that the sets used to approximate the linearization error enclose the exact ones $\left(\overline{\mathcal{L}}^{d} \supseteq \mathcal{L}^{d}\right.$ and $\left.\overline{\mathcal{L}}^{a} \supseteq \mathcal{L}^{a}\right)$. In order to overapproximate the set of linearization errors, we first have to reconstruct the reachable set for all variables $\overline{\mathcal{R}}\left(\tau_{k}\right)$ from the reachable set of the differential variables $\overline{\mathcal{R}}^{d}\left(\tau_{k}\right)$.

\section{A. Reachable Set of Differential and Algebraic Variables}

For a concise notation of the combined reachable set $\overline{\mathcal{R}}\left(\tau_{k}\right)$, we introduce the matrix of generators $G=\left[\begin{array}{lll}g^{(1)} & \ldots g^{(p)}\end{array}\right]$ and the alternative short form of a zonotope $\mathcal{Z}$ as $\mathcal{Z}=(c, G)$, with center $c$ and the matrix of generators $G$.

\section{Proposition 1 (Differential-Algebraic Reachable Set)}

Suppose $\overline{\mathcal{R}}^{d}\left(\tau_{k}\right)=\left(c^{d}, G^{d}\right), \overline{\mathcal{U}}=\left(c^{u}, G^{u}\right), \overline{\mathcal{L}}^{a}=\left(c^{l}, G^{l}\right)$. An overapproximation for the complete reachable set for the differential and algebraic variables is

$$
\overline{\mathcal{R}}\left(\tau_{k}\right)=\left(\left[\begin{array}{c}
c^{d} \\
c^{a}
\end{array}\right],\left[\begin{array}{ccc}
G^{d} & 0 & 0 \\
-F^{-1} D G^{d} & -F^{-1} E G^{u} & -F^{-1} G^{l}
\end{array}\right]\right),
$$

where $c^{a}=y^{*}-F^{-1}\left(g\left(z^{*}\right)+D\left(c^{d}-x^{*}\right)+E\left(c^{u}-u^{*}\right)+c^{l}\right)$, and $\mathbf{0}$ is a matrix of zeros of proper dimension.

Proof: Using (7), the state of the differential-algebraic system is bounded by

$$
\begin{aligned}
{\left[\begin{array}{l}
x(t) \\
y(t)
\end{array}\right] \in } & {\left[\begin{array}{c}
x^{*} \\
y^{*}-F^{-1} g\left(z^{*}\right)
\end{array}\right]+\left[\begin{array}{c}
I \\
-F^{-1} D
\end{array}\right] \Delta x(t) } \\
& +\left[\begin{array}{c}
\mathbf{0} \\
-F^{-1} E
\end{array}\right] \Delta u(t) \oplus\left[\begin{array}{c}
\mathbf{0} \\
-F^{-1}
\end{array}\right] \overline{\mathcal{L}}^{a} .
\end{aligned}
$$

Inserting $\Delta x\left(\tau_{k}\right) \in\left(c^{d}-x^{*}, G^{d}\right), \Delta u\left(\tau_{k}\right) \in\left(c^{u}-u^{*}, G^{u}\right)$, $\overline{\mathcal{L}}^{a}\left(\tau_{k}\right)=\left(c^{l}, G^{l}\right)$ into the above equation yields the proposed computation of $\overline{\mathcal{R}}\left(\tau_{k}\right)$ using the addition and multiplication rule of zonotopes $\mathcal{Z}=(c, G): M \otimes \mathcal{Z}=(M c, M G), \mathcal{Z}_{1} \oplus$ $\mathcal{Z}_{2}=\left(c_{1}+c_{2},\left[\begin{array}{ll}G_{1} & G_{2}\end{array}\right]\right)$.

Note that Proposition 1 is tighter than the Cartesian product $\overline{\mathcal{R}}^{d}\left(\tau_{k}\right) \times \overline{\mathcal{R}}^{a}\left(\tau_{k}\right)$ because the latter result has $\tilde{p}$ more generators, where $\tilde{p}$ is the number of generators of $\overline{\mathcal{R}}^{d}\left(\tau_{k}\right)$. Next, the set of all variables $\overline{\mathcal{R}}\left(\tau_{k}\right)$ is used to overapproximate the set of linearization errors.

\section{B. Bounding the Lagrange Remainder}

We first show the computation of the linearization error $\mathcal{L}^{d}$ and then generalize to $\mathcal{L}$. As described in (5),

$$
\mathcal{L}^{d} \subseteq \frac{1}{2}\left\{\sigma \mid \sigma_{i}=\nu^{T} H^{d,(i)}(\xi) \nu, \xi \in \overline{\mathcal{R}}^{z}, \nu \in \overline{\mathcal{R}}_{\Delta}^{z}\right\}
$$

where we replaced the yet unknown sets $\mathcal{R}^{z}$ and $\mathcal{R}_{\Delta}^{z}$ by the more conservative overapproximations $\overline{\mathcal{R}}^{z}$ and $\overline{\mathcal{R}}_{\Delta}^{z}$.

In order to compute the set of linearization errors, we first compute the possible values of the second derivative $\mathcal{H}^{d,(i)}:=\left\{H^{d,(i)}(\xi) \mid \xi \in \overline{\mathcal{R}}^{z}\right\}$. This is done by first computing the enclosing box $\mathcal{I}=\operatorname{box}\left(\overline{\mathcal{R}}^{z}\right)$, which is obtained as in [15]. Each element of the matrices $H^{d,(i)}(\xi)$ is evaluated for $\xi \in \mathcal{I}$ using interval arithmetic [18].

We present a new technique to compute the set of linearization errors by overapproximating (14) with

$$
\mathcal{L}^{d} \subseteq \frac{1}{2}\left\{\sigma \mid \sigma_{i}=\nu^{T} \otimes \mathcal{H}^{d,(i)} \otimes \nu, \nu \in \overline{\mathcal{R}}_{\Delta}^{z}\right\}
$$

and introducing an overapproximation of a quadratic map:

Lemma 1 (Quadratic Map) Given a zonotope $\mathcal{Z}=(c$, $\left.g^{(1)}, \ldots, g^{(p)}\right)$ and a discrete set of matrices $Q^{(i)} \in \mathbb{R}^{n \times n}$, $i=1 \ldots n$, the set

$$
\mathcal{Z}_{Q}=\left\{\varphi \mid \varphi_{i}=x^{T} Q^{(i)} x, x \in \mathcal{Z}\right\}
$$

is overapproximated by a zonotope

$$
\operatorname{quad}(Q, \mathcal{Z}):=\left(d, h^{(1)}, \ldots, h^{(\sigma)}\right)
$$

with $\sigma=\left(\begin{array}{c}p+2 \\ 2\end{array}\right)-1$ generators, the center $d_{i}=c^{T} Q^{(i)} c+$ $0.5 \sum_{s=1}^{p} g^{(s)^{T}} Q^{(i)} g^{(s)}$ and the generators

$$
\begin{aligned}
j & =1 \ldots p: & h_{i}^{(j)} & =c^{T} Q^{(i)} g^{(j)}+g^{(j)^{T}} Q^{(i)} c \\
j & =1 \ldots p: & h_{i}^{(p+j)} & =0.5 g^{(j)^{T}} Q^{(i)} g^{(j)} \\
l & =\sum_{j=1}^{p-1} \sum_{k=j+1}^{p} 1: & h_{i}^{(2 p+l)} & =g^{(j)^{T}} Q^{(i)} g^{(k)}+g^{(k)^{T}} Q^{(i)} g^{(j)}
\end{aligned}
$$

The complexity of constructing this zonotope overapproximation with respect to the dimension $n$ is $\mathcal{O}\left(n^{5}\right)$.

The proof of this Lemma can be found in [4]. The above Lemma is used in the proof of the following Theorem to overapproximate $\mathcal{L}^{d}$ in (15):

Theorem 1 (Linearization Error) Let each $\mathcal{H}^{d,(i)}$ be bounded by an interval matrix, which we separate to 
$\mathcal{H}^{d}=H_{c}^{d} \oplus\left[-H_{\Delta}^{d}, H_{\Delta}^{d}\right]$, where $H_{c}^{d}, H_{\Delta}^{d} \in \mathbb{R}^{n \times n}$. The linearization error according to (15) is overapproximated by

$$
\begin{aligned}
\mathcal{L}^{d} & \subseteq \frac{1}{2}\left\{z^{T} \mathcal{H}^{d,(i)} z \mid z \in \mathcal{R}_{\Delta}^{z}\right\} \\
\subseteq & \frac{1}{2}\left(\operatorname{quad}\left(H_{c}^{d}, \overline{\mathcal{R}}_{\Delta}^{z}\right) \oplus[-\eta, \eta]\right), \\
& \eta=\left|\overline{\mathcal{R}}_{\Delta}^{z}\right|^{T} H_{\Delta}^{d}\left|\overline{\mathcal{R}}_{\Delta}^{z}\right|, \quad\left|\overline{\mathcal{R}}_{\Delta}^{z}\right|=\left|c_{\Delta}^{z}\right|+\sum_{i=1}^{p}\left|g_{\Delta}^{z,(i)}\right| .
\end{aligned}
$$

The complexity with respect to the dimension $n$ is $\mathcal{O}\left(n^{5}\right)$.

Proof: Negligence of dependencies results in overapproximations such that we have

$$
\begin{aligned}
\mathcal{L}^{d} & \subseteq \frac{1}{2}\left\{z^{T} \mathcal{H}^{d,(i)} z \mid z \in \mathcal{R}_{\Delta}^{z}\right\} \\
\subseteq & \frac{1}{2}(\underbrace{\left\{z^{T} H_{c}^{d,(i)} z \mid z \in \mathcal{R}_{\Delta}^{z}\right\}}_{=\operatorname{quad}\left(H_{c}^{d}, \overline{\mathcal{R}}_{\Delta}^{z}\right)} \\
\oplus & \underbrace{\left\{z^{T}\left[-H_{\Delta}^{d,(i)}, H_{\Delta}^{d,(i)}\right] z \mid z \in \mathcal{R}_{\Delta}^{z}\right\}}_{\subseteq\left(\mathcal{R}_{\Delta}^{z}\right)^{T} \otimes\left[-H_{\Delta}^{d,(i)}, H_{\Delta}^{d,(i)}\right] \otimes \mathcal{R}_{\Delta}^{z}})
\end{aligned}
$$

Given $N \in \mathbb{R}^{q \times m}$ and an interval matrix $\mathcal{S}=[-S, S]$ with symmetric bound $S \in \mathbb{R}^{l \times q}$, we have that $\mathcal{S} N=$ $[-S|N|, S|N|]$, see [3]. From this follows for a zonotope $\mathcal{R}_{\Delta}^{z}=\left\{c \oplus \bigoplus_{i=1}^{p}[-1,1] g_{\Delta}^{z,(i)}\right\}$ that

$\left[-H_{\Delta}^{d,(i)}, H_{\Delta}^{d,(i)}\right] \otimes \mathcal{R}_{\Delta}^{z}$

$\subseteq\left(\left[-H_{\Delta}^{d,(i)}, H_{\Delta}^{d,(i)}\right] \otimes c_{\Delta}^{z}\right) \oplus\left(\bigoplus_{i=1}^{p}\left[-H_{\Delta}^{d,(i)}, H_{\Delta}^{d,(i)}\right] \otimes g_{\Delta}^{z,(i)}\right)$ $=[-H_{\Delta}^{d,(i)} \underbrace{\left(\left|c_{\Delta}^{z}\right|+\sum_{i=1}^{p}\left|g_{\Delta}^{z,(i)}\right|\right)}_{=\left|\overline{\mathcal{R}}_{\Delta}^{z}\right|}, H_{\Delta}^{d,(i)} \underbrace{\left(\left|c_{\Delta}^{z}\right|+\sum_{i=1}^{p}\left|g_{\Delta}^{z,(i)}\right|\right)}_{=\left|\overline{\mathcal{R}}_{\Delta}^{z}\right|}]$.

Thus, $\left(\mathcal{R}_{\Delta}^{z}\right)^{T} \otimes\left[-H_{\Delta}^{d,(i)}, H_{\Delta}^{d,(i)}\right] \otimes \mathcal{R}_{\Delta}^{z} \subseteq[-\eta, \eta]$, where $\eta$ is as specified in the Theorem.

Since all other operations of the reachable set computation, such as linear maps, Minkowski addition, convex hull, and so on are at most $\mathcal{O}\left(n^{3}\right)$, the overall complexity of the reachability analysis is $\mathcal{O}\left(n^{5}\right)$ due to the complexity of the linearization error computation in Theorem 1 (when splitting is not required). It remains to compute the linearization error $\mathcal{L}$ in (8) using the techniques presented above.

Corollary 1 (Linearization Error $\mathcal{L}$ ) Given the zonotopes $\mathcal{L}_{c}^{d}=\operatorname{quad}\left(H_{c}^{d}, \overline{\mathcal{R}}_{\Delta}^{z}\right)=(d, H)$ and $\mathcal{L}_{c}^{a}=\operatorname{quad}\left(H_{c}^{a}, \overline{\mathcal{R}}_{\Delta}^{z}\right)=$ $(e, V)$, the overapproximative set of linearization errors is computed as

$$
\begin{gathered}
\mathcal{L}=\underbrace{\frac{1}{2}\left(d-C F^{-1} e, H-C F^{-1} V\right)}_{=: \mathcal{L}_{c}} \\
\underbrace{\frac{1}{2}\left([-\zeta, \zeta] \oplus\left(-C F^{-1}\right)[-\varrho, \varrho]\right)}_{=: \mathcal{L}_{\Delta}}, \\
\zeta=\left|\mathcal{R}_{\Delta}^{z}\right|^{T} H_{\Delta}^{d}\left|\mathcal{R}_{\Delta}^{z}\right|, \quad \varrho=\left|\mathcal{R}_{\Delta}^{z}\right|^{T} H_{\Delta}^{a}\left|\mathcal{R}_{\Delta}^{z}\right| .
\end{gathered}
$$

Due to space limitations we omit the proof.

The overapproximated linearization error $\mathcal{L}$ is used to obtain the final result by replacing the set of uncertain inputs $\overline{\mathcal{U}}$ with $\tilde{\mathcal{U}}:=k \oplus \tilde{B}\left(\mathcal{U} \oplus\left(-u^{*}\right)\right) \oplus \mathcal{L}$ (see (10)), which is used to compute $\mathcal{R}_{p}^{d}(r)$ according to (12). We finally obtain $\mathcal{R}^{d}\left(\tau_{k}\right)=$ $\mathcal{R}_{\text {affine }}^{d}\left(\tau_{k}\right) \oplus \mathcal{R}_{p}^{d}(r)$ and analogously the overapproximations for the reachable set at points in time, where $\mathcal{R}_{\text {affine }}^{d}\left(\tau_{k}\right)$ is taken from (13). Based on $\mathcal{R}^{d}\left(\tau_{k}\right)$, the algebraic reachable set $\mathcal{R}^{a}\left(\tau_{k}\right)$ is computed by evaluating the algebraic part of Prop. 1. The complete approach is applied to the power system example in the next section.

\section{NUMERICAL RESULT}

The considered verification task is to show that after a power drop-out of a power plant and its subsequent reconnection to the grid, the system state returns to its original operating point. We show this for a set of initial states by computing the reachable set of the differential variables until it is enclosed by the initial set again. We first present the mathematical model of the power system and then show the results of the reachability analysis.

\section{A. Mathematical Model}

We use the IEEE 14-bus benchmark system enhanced by generator dynamics, which is depicted in Fig. 4. In order to obtain the correct equations for the relatively complex 14bus system, we auto-generate the equations using symbolic computations in MATLAB. First, the power flow equations are obtained according to [24] for each bus, where variable indices refer to the bus number.

The absolute value of the voltage is denoted by $\left|V_{i}\right|$ [p.u] (p.u.: per unit), the angle of the voltage by $\delta_{i}$ [rad], the active power by $P_{i}$ [p.u.], and the reactive power by $Q_{i}$ [p.u.], where inflow of power is positive. The buses are connected via admittances $Y_{i j}$, whose absolute value and angle are denoted by $\left|Y_{i j}\right|$ and $\Psi_{i j}=\angle Y_{i j}$. We denote the generator production by $P_{g, i}, Q_{g, i}$, the demand by $P_{d, i}, Q_{d, i}$, the generator voltage by $E_{i}$ [p.u.], the generator phase angle by $\tilde{\delta}_{i}$ [rad], and the admittance from the generator to the $i^{\text {th }}$ generator bus by $Y_{g, i}$, where $\Psi_{g, i}=\angle Y_{g, i}$ [rad]. The generator phase angles $\delta_{i}=\tilde{\delta}_{i}-\tilde{\delta}_{1}$ and the bus phase angles $\Theta_{i}=\tilde{\Theta}_{i}-\tilde{\delta}_{1}$ are relative to $\tilde{\delta}_{1}$ so that the generated power of the slack bus and generator buses $\left(i=1 \ldots N_{g}\right)$ are (see [24])

$$
\begin{aligned}
P_{g, i} & =E_{i} V_{i}\left|Y_{g, i}\right| \cos \left(\Psi_{g, i}+\delta_{i}-\Theta_{i}\right)-V_{i}^{2}\left|Y_{g, i}\right| \cos \left(\Psi_{g, i}\right), \\
Q_{g, i} & =-E_{i} V_{i}\left|Y_{g, i}\right| \sin \left(\Psi_{g, i}+\delta_{i}-\Theta_{i}\right)+V_{i}^{2}\left|Y_{g, i}\right| \sin \left(\Psi_{g, i}\right) .
\end{aligned}
$$

The power flow equations as in [24, p.174] of each bus are

$$
\begin{aligned}
P_{i} & =P_{g, i}+P_{d, i}=\sum_{j=1}^{N_{g}+N_{l}} V_{i} V_{j}\left|Y_{i j}\right| \cos \left(\Psi_{i j}+\Theta_{j}-\Theta_{i}\right), \\
Q_{i}=Q_{g, i}+Q_{d, i} & =-\sum_{j=1}^{N_{g}+N_{l}} V_{i} V_{j}\left|Y_{i j}\right| \sin \left(\Psi_{i j}+\Theta_{j}-\Theta_{i}\right) .
\end{aligned}
$$

The dynamic equations are described by a generator model [13]. For simplicity, we use the same model for all generators 
and synchronous condensers. The variables of the $i^{\text {th }}$ generator are the voltage angle $\delta_{i}[\mathrm{rad}]$, the angular velocity $\omega_{i}[\mathrm{rad} / \mathrm{s}]$, and the torque $T_{m, i}$ [p.u.], and the commanded powers $P_{c, i}$ [p.u.]:

$$
\begin{aligned}
\dot{\delta}_{i} & =\omega_{i}-\omega_{1} \\
\dot{\omega}_{i} & =-\frac{D_{i}}{M_{i}}\left(\omega_{i}-\omega_{1}\right)+\frac{1}{M_{i}} T_{m, i}-\frac{1}{M_{i}} P_{g, i} \\
\dot{T}_{m, i} & =-\frac{1}{T_{S V, i} R_{D, i} \omega_{s}}\left(\omega_{i}-\omega_{s}\right)-\frac{1}{T_{S V, i}} T_{m, i}+\frac{1}{T_{S V, i}} P_{c, i},
\end{aligned}
$$

where $M_{i}\left[\mathrm{MJ} / \mathrm{Hz}^{2}\right]$ is the rotational inertia, $D_{i}[\mathrm{~s} / \mathrm{rad}]$ the damping coefficient, $T_{S V, i}[\mathrm{~s}]$ is the time constant of the governor, and $\frac{1}{R_{D, i}}[-]$ is the proportional gain of the governor. For $i=1$, the dynamics is solely described by $\omega$ and $T_{m}$ since the phase angle is always 0 .

The power drop-out of the $i^{\text {th }}$ power plant is modeled by setting the active and reactive power in (16) and (17) to zero $\left(P_{g, i}=0, Q_{g, i}=0\right)$. When the $i^{\text {th }}$ power plant is not on the grid, the variable $E_{i}$ is removed from (16), (17), and is no longer an unknown variable. The generator parameters are listed in Tab. I and the one of the IEEE 14-bus system in [27].

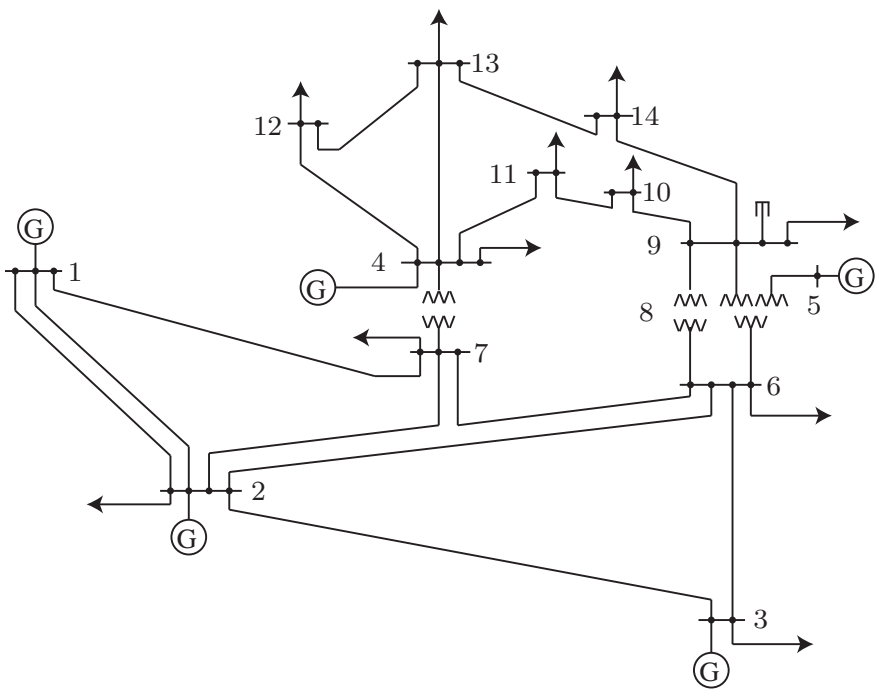

Fig. 4. IEEE 14-bus benchmark system.

TABLE I

PARAMETERS OF THE GENERATORS.

\begin{tabular}{cccccccc}
\hline$\forall i:$ & $M_{i}$ & $D_{i}$ & $\left|Y_{g, i}\right|$ & $\Psi_{g, i}$ & $T_{S V, i}$ & $R_{D, i}$ & $\omega_{s}$ \\
& $\frac{1}{15 \pi}$ & 0.04 & 5 & $-\frac{\pi}{2}$ & 1 & 0.05 & $120 \pi$ \\
\hline
\end{tabular}

\section{B. Reachability Analysis}

We investigate the transient stability by a power drop-out of the largest power plant at bus 1 . The power system is in normal operation for the first time interval $t=[0,0.1]$ [s], which we call pre-fault phase. In the time interval $t=[0.1,0.13][\mathrm{s}]$, the power plant at bus 1 producing the most power is taken off the grid, which we refer to as the fault-on phase. At $t=$ $0.13[\mathrm{~s}]$, the power plant is reconnected, which starts the postfault phase. The reachable set computation is stopped when the reachable set of differential variables is enclosed by the initial set of states, proving that all differential state variables return to the original operating point (steady state). We choose the set of initial states for all $i$ as: $\delta_{i}=\delta_{i}^{0} \oplus 0.01 \cdot[-1,1]$, $\omega_{i}=\omega_{i}^{0} \oplus 0.1 \cdot[-1,1], T_{m, i}=T_{m, i}^{0} \oplus 0.001 \cdot[-1,1]$, where the superscripted zero refers to the steady state solution.

The reachable sets for different projections onto differential and algebraic variables is shown in Fig. 5 and Fig. 6. The simulations of system trajectories from randomly chosen initial states are indicated by black lines. Note that the algebraic values jump when the power plant is taken off the grid and when it is reconnected to the grid. At time $t=4.32$ [s], the initial set is reached after 540 iterations, which took 3889 [s] to compute in MATLAB on an i7 Processor and 6GB memory.

We are not able to compare the obtained reachable sets with other methods, since none of the previous work on systems with DAEs would scale to the size of the problem presented here.
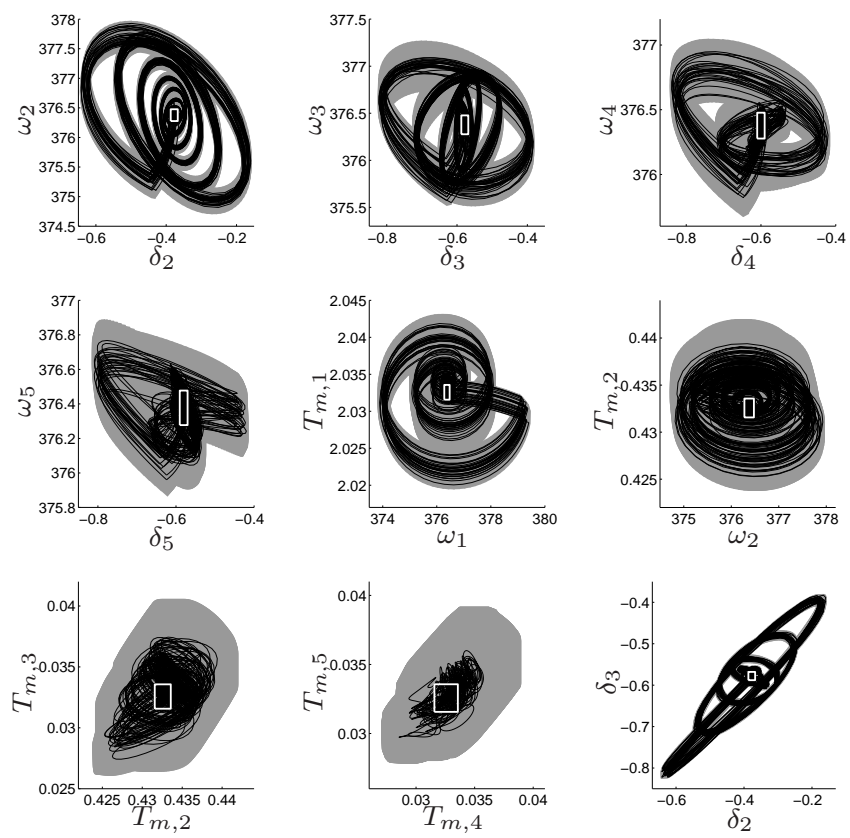

Fig. 5. Reachable set of selected projections of differential variables. Black lines show random simulations, the gray area shows the reachable set, and the white box the initial set.

\section{CONCLUSION}

We present an approach for computing the set of all (infinitely many) transient responses of power systems for a set of initial states, which makes it possible to prove that all responses returns to the set of initial states. The presented approach is versatile since it can handle any nonlinear differential-algebraic equations with index-1 property. If not all responses return to the set of initial states, one obtains feedback for system corrections by investigating how the reachable set evolved. When using Lyapunov methods one has no such feedback since Lyapunov methods may fail because 

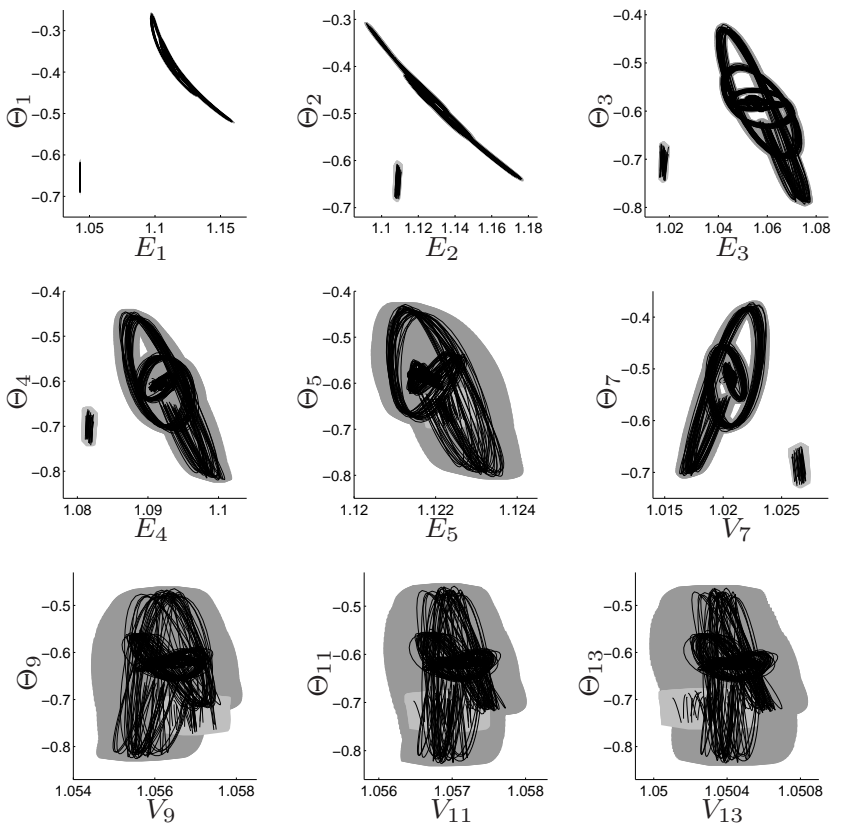

Fig. 6. Reachable set of selected projections of algebraic variables. Black lines show random simulations, the dark gray area shows the reachable set during pre-fault and post-fault operation, and the light gray area shows the reachable set during fault-on operation.

one cannot find a proper Lyapunov function, or because the system is indeed unstable.

Computing reachable sets of differential-algebraic systems of practically relevant size was previously infeasible, but the new computational techniques provided in this work with complexity $\mathcal{O}\left(n^{5}\right)$ with respect to the system dimension $n$ (when splitting is not required), shows that reachability analysis might become a useful tool for power system engineers. In addition to the presented transient stability problem, the same approach can be readily used to compute the system response to uncertainties in the power production, which is caused by e.g. wind turbines. It is also possible to integrate parametric uncertainties into the power system, which would require some extensions to the current algorithm as presented in [5].

\section{ACKNOWLEDGMENT}

This research was supported in part by U.S. National Science Foundation grant number CCF-0926181.

\section{REFERENCES}

[1] L. F. C. Alberto, F. H. J. R. Silva, and N. G. Bretas. Direct methods for transient stability analysis in power systems: State of art and future perspectives. In Proc. of the IEEE Porto Power Tech Conference, 2001.

[2] G. Aloisio, M. A. Bochicchio, M. La Scala, and R. Sbrizzai. A distributed computing approach for real-time transient stability analysis. IEEE Transactions on Power Systems, 12(2):981-987, 1997.

[3] M. Althoff. Reachability Analysis and its Application to the Safety Assessment of Autonomous Cars. Dissertation, Technische Universität München, 2010. http://nbnresolving.de/urn/resolver.pl?urn:nbn:de:bvb:91-diss-20100715-963752$1-4$.

[4] M. Althoff and B. H. Krogh. Avoiding geometric intersection operations in reachability analysis of hybrid systems. In Hybrid Systems: Computation and Control, pages 45-54, 2012.
[5] M. Althoff, B. H. Krogh, and O. Stursberg. Modeling, Design, and Simulation of Systems with Uncertainties, chapter Analyzing Reachability of Linear Dynamic Systems with Parametric Uncertainties, pages 69-94. Springer, 2011.

[6] M. Althoff, C. Le Guernic, and B. H. Krogh. Reachable set computation for uncertain time-varying linear systems. In Hybrid Systems: Computation and Control, pages 93-102, 2011.

[7] M. Althoff, O. Stursberg, and M. Buss. Reachability analysis of nonlinear systems with uncertain parameters using conservative linearization. In Proc. of the 47th IEEE Conference on Decision and Control, pages 4042-4048, 2008.

[8] P. M. Anderson and A. Bose. A probabilistic approach to power system stability analysis. IEEE Transactions on Power Apparatus and Systems, 102(8):2430-2439, 1983.

[9] A. G. Bahbah and A. A. Girgis. New method for generators angles and angular velocities prediction for transient stability assessment of multimachine power systems using recurrent artificial neural network. IEEE Transactions on Power Systems, 19(2):1015-1022, 2004.

[10] R. C. Bergvall and P. H. Robinson. Quantitative mechanical analysis of power system transient disturbances. Transactions of the American Institute of Electrical Engineers, 47(3):915-925, 1928.

[11] M. Berz and G. Hoffstätter. Computation and application of Taylor polynomials with interval remainder bounds. Reliable Computing, 4:8397, 1998.

[12] H.-D. Chang, C.-C. Chu, and G. Cauley. Direct stability analysis of electric power systems using energy functions: Theory, applications, and perspective. Proceedings of the IEEE, 83(11):1497-1529, 1995

[13] Y. C. Chen and A. D. Domínguez-García. Assessing the impact of wind variability on power system small-signal reachability. In Proc. of the International Conference on System Sciences, pages 1-8, 2011.

[14] G. Frehse, C. Le Guernic, A. Donzé, S. Cotton, R. Ray, O. Lebeltel, R. Ripado, A. Girard, T. Dang, and O. Maler. SpaceEx: Scalable verification of hybrid systems. In Proc. of the 23rd International Conference on Computer Aided Verification, LNCS 6806, pages 379395. Springer, 2011.

[15] A. Girard. Reachability of uncertain linear systems using zonotopes. In Hybrid Systems: Computation and Control, LNCS 3414, pages 291-305. Springer, 2005.

[16] A. Girard and C. Le Guernic. Efficient reachability analysis for linear systems using support functions. In Proc. of the 17th IFAC World Congress, pages 8966-8971, 2008.

[17] A. Girard, C. Le Guernic, and O. Maler. Efficient computation of reachable sets of linear time-invariant systems with inputs. In Hybrid Systems: Computation and Control, LNCS 3927, pages 257-271. Springer, 2006.

[18] L. Jaulin, M. Kieffer, and O. Didrit. Applied Interval Analysis. Springer, 2006.

[19] L. Jin, H. Liu, R. Kumar, J. D. McCalley, N. Elia, and V. Ajjarapu. Power system transient stability design using reachability based stabilityregion computation. In Proc. of the 37th Annual North American Power Symposium, pages 338-343, 2005.

[20] G. Lafferriere, G. J. Pappas, and S. Yovine. Symbolic reachability computation for families of linear vector fields. Symbolic Computation, 32:231-253, 2001.

[21] M. Moechtar, T. C. Cheng, and L. Hu. Transient stability of power system - a survey. In Proc. of the WESCON conference, pages 166$171,1995$.

[22] D. R. Ostojić and G. T. Heydt. Transient stability assessment by pattern regognition in the frequency domain. IEEE Transactions on Power Systems, 6(1):231-237, 1991.

[23] M. Ribbens-Pavella and F. J. Evans. Direct methods for studying dynamics of large-scale electric power systems - a survey. Automatica, 21(1):1-21, 1985.

[24] P. Schavemaker and L. van der Sluis. Electrical Power System Essentials. Wiley, 2008.

[25] J. Shu, W. Xue, and W. Zheng. A parallel transient stability simulation for power systems. IEEE Transactions on Power Systems, 20(4):17091717, 2005.

[26] Y. Susuki, T. Sakiyama, T. Ochi, T. Uemura, and T. Hikihara. Verifying fault release control of power system via hybrid system reachability. In Proc. of the 40th North American Power Symposium, 2008.

[27] University of Washington. Power systems test case archive. http://www. ee.washington.edu/research/pstca/. 